\title{
DESIGN METHODOLOGY OF SPACE SYSTEMS CONTROL COMPLEX OPTIMIZED STRUCTURE
}

\author{
Mehran Mirshams \\ K. N. Toosi University of Technology \\ Tehran, Iran \\ Mirshams@kntu.ac.ir \\ Asghar Ebrahimi \\ M. A. University of Technology \\ Tehran, Iran \\ ebrahimi@mut.ac.ir \\ Alireza Ahmadi ${ }^{*}$ \\ K. N. Toosi University of Technology \\ Tehran, Iran \\ $\underline{\text { Ali.r.ahmadi@gmail.com }}$
}

\begin{abstract}
After launch, access to space segment (the satellite) and space operations can be done only by means of the ground segment and its radio link between satellite and TT\&C ground stations; so the preliminary activities must be fulfilled before. In this article, the three main methods of satellite control during flight have been introduced and the optimum method has been concluded. The main methods of satellite control are: command-based, time schedule - based and coordinate - based control method; the other methods are combinations of these three methods. In order to determine the optimum one, the AHP (Analytic Hierarchy Process) method has been used. All the satellite control methods have advantages and disadvantages so the mission planners and system engineers will select the best, according to the criteria and the weight of each criterion. The satellite onboard computer (OBC) will be programmed based on the selected control method. The supposed criteria in this article are: fastness (the time interval between decision making and start-up), accuracy, cost, onboard power consumption and reliability. Among the introduced methods, the combination of command - based and time schedule - based control method has been selected as the optimum.
\end{abstract}

Keywords: space systems, control, command, time schedule, coordinate, AHP, optimum.

\begin{tabular}{|c|c|}
\hline $\mathbf{n}$ & Nu mber of criteria \\
\hline $\mathbf{W}_{\mathbf{n}}$ & Weight of a criterion \\
\hline $\mathbf{W}_{\mathbf{n}}$ & Percent weight of a criteria \\
\hline $\mathbf{m}$ & Nu mber of methods \\
\hline $\mathbf{W}_{\mathbf{m n}}$ & $\begin{array}{l}\text { Re lative weight of each method based on } \\
\text { each criteria }\end{array}$ \\
\hline $\mathbf{W}_{\mathbf{m n}}$ & $\begin{array}{l}\text { Relative percent weight of each method } \\
\text { based on each criteria }\end{array}$ \\
\hline $\mathbf{U}_{\mathbf{m}}$ & Combined weight of each method \\
\hline
\end{tabular}

${ }^{*}$ Corresponding author 


\section{Introduction}

After launch, access to space segment (the satellite) and space operations can be done only by means of the ground segment and its radio link between satellite and TT\&C ground stations; so the preliminary activities must be fulfilled before [1]. In order to control the satellite during the flight, there are three main methods that will be introduced and compared to each other. The other methods are resulted from combination of the main ones [2]. All the satellite control methods have advantages and disadvantages so the mission planners and system engineers will select the best according to the criteria and the weight of each criterion [3]. The satellite onboard computer (OBC) will be programmed based on the selected control method [4].

The main control methods of satellites are:

- Command - based control method

- Time schedule - based control method

- Coordinate - based control method

And the combined ones are as below:

- Command - time schedule based control method

- Command - coordinate based control method

- Time schedule - coordinate control method [5] , [3]

This article focused on small class of remote sensing satellites.

\subsection{Command - Based Control Method}

In this method, all standard activities on the satellite will be executed just according to the commands received from the TT\&C ground stations. The ground control center determines these commands and then sends them to the satellite by means of the TT\&C ground stations [6].

\subsection{Time schedule - based control method}

In this method, mission operations plan and the other adjustments are generated as a function of time. This digital time table will be installed on the satellite onboard computer. The most important requirement for implementation of this method is generation of the plan and on time transmission to the OBC. The contents of the plan are satellite subsystems status, their components changes and these changes duration. Switching to on and off status in sensors of a remote sensing satellite is an example of these changes. In terms of the time interval between decision making and start up and also accuracy, this method is ranked in low level. Some of the reasons are as below:

1. In most cases, telemetry data is received in GCC and analyzed with some delays.

2. The time delay between command determination and its transmission is too long.

Despite these disadvantages, time schedule - based was one of the most common control methods. The implementation of this method is fulfilled as bellow:

- The logical and computational tasks will be done in GCC.

- The results will be transmitted to the satellite to be executed onboard as a function of time.

Upon the above descriptions, it is obvious that why this method is named time schedule - based.

In remote sensing satellites miss ion, the ultimate goal is quality of the images. Based on this criterion, the capacity of the sensors may not be completely used because of errors in satellite position predictions. In order to determine the exact time of image acquisition (the exact time of switching on the satellite sensors), the satellite trajectory must be predicted as exact as possible. Upon this prediction, the approximate time that the satellite will be over the right point to capture image, may be determined. Due 
to the inaccuracies in the above mentioned prediction the margins may be too long in order to completely cover the area. But these margins are not useful for image users; they will waste the limited source of satellite power and will lessen the Power Supply Subsystem efficiency [6].

\subsection{Coordinate - based control method}

In coordinate - based control method, each section of space operations is a function of satellite coordinate and its orbital position. In order to use this control method, there must be an orbital parameters or coordinate determiner onboard the satellite to determine the coordinate of satellite center of mass in each moment of time. The OBC will generate the control command of onboard subsystems. This command may change the status of the corresponding subsystem by comparison of the target area and the current coordinate of the satellite. The satellites which the coordinate - based method has been used in them are more independent than the others.

The advantages of this control method are:

a. The image acquisition operation unlike the time schedule - based method, will be done upon the plan that has been generated by the $\mathrm{OBC}$ and not the ground control center (independency) and this program is a function of satellite orbital coordinate in comparison the target area.

The more accurate satellite coordinate determination will result the least margins to completely cover the target area, then the power consumption will be reduced and the spatial resolution of images will be increased.

b. As mentioned above, using this method entails the accurate satellite coordinate determination in every moment of time so there must be a navigation system onboard the satellite like GPS or GLONASS.

c. The coordinate - based method is a state of the art method and the needed equipments for implementing of such a method are more expensive than the others. In order to reduce the cost of this method implementation, the following recommendations in design and manufacturing the satellite will be useful:

- The two most important parameters that must be under control in remote sensing satellites are orbital coordinate and attitude; so in design of satellite positioning and ADC (Attitude Determination and Control) Subsystems, the multipurpose devices must be preferred to determine the satellite position and attitude simultaneously. In addition to cost, also the weight will become lower too.

- The ratio of image acquisition time to the orbital period of a remote sensing satellite is about $10-15 \%$. In order to reduce the power consumption, there must be a less accurate positioning and attitude determination subsystem onboard, besides the more accurate ones. The accurate subsystem will be switched on just when the satellite is in the image acquisition mode and in the other modes the other one may be on.

d. Other than the onboard determination of satellite coordinate method, the orbital parameters may be determined by ranging, observatory or surveillance ground stations. But in order to use this method, there must be an adequate number of stations with a proper configuration to perform enough measurements in a short interval of time.

In order to implement the coordinate - based control method, the following requirements must be provided:

- The attitude determination error must be less than 10 angular min in yaw, pitch and roll orientations.

- The RMS (Root Mean Square) error of positioning must be less than $100 \mathrm{~m}$ in height, $5 \mathrm{Km}$ in direction of motion and less than $200 \mathrm{~m}$ in perpendicular direction to the motion. The required data to calculate the RMS Error must be obtained from about 5-8 cycle measurements. 
Essentially because of its independency level, in this method the main element of satellite control during flight is $\mathrm{OBC}[8]$.

\subsection{The coor di nate - time schedule based satellite control method}

In this kind of satellite control method, the control commands will not be generated upon the measurable but predictable parameters of the satellite center of mass position. This method has two main advantages. It may reduce the volume of the current data in Automatic Control System and make it possible to control the satellite in the orbit sections where there is no access to it by the ground control segment. In this method the satellite position prediction software will be loaded in the OBC [9].

\section{The optimum satellite control method determination based on qualitative parameters}

So far it seems that the time schedule - based control method in combination with command control method will be the optimum one. Although the time schedule - based compared with the coordinate based is a time consuming control method but since it provides the maximum independency to the ground control segment personnel, it is more prevalent than the others [10].

The required information to compare these methods has been gathered in the table 1 .

Table 1: The optimum satellite control method determination upon the modal criteria

\begin{tabular}{|c|c|c|c|}
\hline No. & Decision Making Criteria & $\begin{array}{r}\text { Comparison resul } \\
\text { (high }- \text { medium - l }\end{array}$ & \\
\hline \multirow{5}{*}{1} & \multirow{5}{*}{ fastness (the time interval between decision making and start-up) } & Command & $\mathrm{L}$ \\
\hline & & Time schedule & $\mathrm{L}$ \\
\hline & & Coordinate & $\mathrm{H}$ \\
\hline & & Time + coordinate & $\overline{\mathrm{H}}$ \\
\hline & & Command + time & $\mathrm{M}$ \\
\hline \multirow{5}{*}{2} & \multirow{5}{*}{ Accuracy of control commands execution } & Command & $\overline{\mathrm{M}}$ \\
\hline & & Time schedule & $\mathrm{L}$ \\
\hline & & Coordinate & $\mathrm{H}$ \\
\hline & & Time + coordinate & $\mathrm{M}$ \\
\hline & & Command + time & $\bar{M}$ \\
\hline \multirow{5}{*}{3} & \multirow{5}{*}{ Ground control segment workload } & Command & $\mathrm{H}$ \\
\hline & & Time schedule & $\mathrm{H}$ \\
\hline & & Coordinate & $\mathrm{L}$ \\
\hline & & Time + coordinate & $\mathrm{L}$ \\
\hline & & Command + time & $\mathrm{M}$ \\
\hline \multirow{5}{*}{4} & \multirow{5}{*}{ Space control segment workload } & Command & $\mathrm{L}$ \\
\hline & & Time schedule & $\mathrm{M}$ \\
\hline & & Coordinate & $\mathrm{H}$ \\
\hline & & Time + coordinate & $\mathrm{H}$ \\
\hline & & Command + time & $\mathrm{M}$ \\
\hline \multirow{5}{*}{5} & \multirow{5}{*}{$\begin{array}{l}\text { Required Onboard control devices: } \\
\text { - Satellite attitude and position determiner } \\
\text { - Satellite attitude and pos ition controller }\end{array}$} & Command & $\mathrm{L}$ \\
\hline & & Time schedule & $\mathrm{L}$ \\
\hline & & Coordinate & $\mathrm{H}$ \\
\hline & & Time + coordinate & $\mathrm{M}$ \\
\hline & & Command + time & $\bar{M}$ \\
\hline
\end{tabular}




\begin{tabular}{|c|c|c|c|}
\hline \multirow{5}{*}{6} & \multirow{5}{*}{$\begin{array}{l}\text { Power consumption during space operations: } \\
\text { - Image margins to complete cover of target area } \\
\text { - Satellite attitude and position accurate control, out of target } \\
\text { area }\end{array}$} & Command & $\mathrm{M}$ \\
\hline & & Time schedule & $\mathrm{H}$ \\
\hline & & Coordinate & $\bar{M}$ \\
\hline & & Time + coordinate & $\bar{M}$ \\
\hline & & Command + time & $\mathrm{M}$ \\
\hline \multirow{5}{*}{7} & \multirow{5}{*}{ Satellite independency during space operations } & Command & $\mathrm{L}$ \\
\hline & & Time schedule & $\mathrm{H}$ \\
\hline & & Coordinate & $\mathrm{H}$ \\
\hline & & Time + coordinate & $\mathrm{H}$ \\
\hline & & Command + time & $\bar{M}$ \\
\hline \multirow{5}{*}{8} & \multirow{5}{*}{$\begin{array}{l}\text { Received images Quality } \\
\text { - Image margins } \\
\text { - Image spatial resolution }\end{array}$} & Command & $\mathrm{M}$ \\
\hline & & Time schedule & $\mathrm{M}$ \\
\hline & & Coordinate & $\mathrm{H}$ \\
\hline & & Time + coordinate & M \\
\hline & & Command + time & $\bar{M}$ \\
\hline
\end{tabular}

\section{The optimum satellite control method determination based on quantitative parameters}

In this article, AHP has been used to determine the optimum satellite control method. The process sequences are as follows [11].

\subsection{Options}

As mentioned above the options are as following:

1. Command - based control method

2. Time schedule - based control method

3. Coordinate - based control method

4. Command and time schedule - based control method

5. Time - coordinate based control method

\subsection{Criteria}

As mentioned in table 1 the criteria are as following:

1. The time interval between decision making and start-up

2. Accuracy of control commands execution

3. Low workload in ground control segment

4. High workload in space control segment

5. The least required onboard accessories

6. Low power consumption during space operations

7. Satellite high independency

8. High quality of received images

9. Cost

In AHP method the criteria must be independent from each other then according to the following points, the independent ones will be selected among the criteria:

- High workload in the space segment means low workload in ground control segment and then the criteria number 3 is deleted.

- High satellite independency and the least required onboard accessories are antonyms then the criteria number 5 is deleted. 
- High workload in the space segment is opposite to the least required onboard accessories so the criteria number 7 is deleted.

- High workload in space control segment means high power consumption during space operations then the first criterion is deleted.

- High quality of received images is one of the aspects of satellite optical payload reliability so the number 8 criterion is deleted and reliability is replaced.

Therefore the remained criteria are as following:

- Fastness

- Accuracy

- Cost

- Power consumption

- Reliability

\subsection{Determination of the weight and percent weight of each criteria}

Since the importance of each criterion is different from the other, in AHP method, the decis ion maker must assign a proprietary weight to each one. These has been indicated as $W_{1}, W_{2}, \ldots, W_{n}$ that $n$ is the number of criteria.

In order to calculate the percent weight, each weight must be divided on the summation of all criteria weights and multiplied by 100 as shown in the equation (1).

$$
w_{n}=\frac{W_{n}}{\sum_{i=1}^{n} W_{i}} \times 100
$$

The calculated weights have been gathered in table 2 .

Table 2. Weight determination of criteria

\begin{tabular}{|c|c|c|c|}
\hline No. & Criteria & Weight & Weight percent \\
\hline 1 & Fastness & 30 & 8.3 \\
\hline 2 & Accuracy & 60 & 16.7 \\
\hline 3 & Cost & 100 & 27.8 \\
\hline 4 & Power consumption & 70 & 19.4 \\
\hline 5 & Reliability & 100 & 27.8 \\
\hline
\end{tabular}

\subsection{Determination of each criterion relative weight}

In this phase, each criterion relative weight matrix must be generated. In table 3 the relative weights has been indicated as a number between 0 and 100 .

Table 3: Matrix of relative weights.

\begin{tabular}{|c|c|c|c|c|c|c|}
\cline { 3 - 7 } \multicolumn{2}{c|}{} & 1 & 2 & 3 & 4 & 5 \\
\cline { 3 - 7 } \multicolumn{2}{c|}{} & Accuracy & Cost & Fastness & Power consumption & Reliability \\
\hline 1 & Time schedule & 40 & 80 & 50 & 80 & 90 \\
\hline 2 & Command & 30 & 90 & 30 & 90 & 95 \\
\hline 3 & Coordinate & 60 & 30 & 80 & 20 & 98 \\
\hline 4 & Command + time & 50 & 80 & 60 & 80 & 93 \\
\hline 5 & Time + coordinate & 70 & 20 & 90 & 20 & 99 \\
\hline
\end{tabular}


In order to calculate the relative weight, the above matrix must be normalized. To normalize the above matrix, each cell must be divided to the summation of the cells in its respective column as indicated in the equation (2).

$$
w_{m n}=\frac{W_{m n}}{\sum_{j=1}^{n} W_{m, j}}
$$

The normalized matrix has been shown in the table 4 .

Table 4: Normalized matrix of weights based on any criterion

\begin{tabular}{|c|c|c|c|c|c|c|}
\cline { 3 - 7 } \multicolumn{2}{c|}{} & 1 & 2 & 3 & 4 & 5 \\
\cline { 3 - 7 } \multicolumn{2}{c|}{} & Accuracy & Cost & Fastness & Power consumption & Reliability \\
\hline 1 & Time schedule & 0.16 & 0.267 & 0.161 & 0.276 & 0.189 \\
\hline 2 & Command & 0.12 & 0.3 & 0.097 & 0.31 & 0.2 \\
\hline 3 & Coordinate & 0.24 & 0.1 & 0.258 & 0.069 & 0.206 \\
\hline 4 & Command + time & 0.2 & 0.267 & 0.194 & 0.276 & 0.196 \\
\hline 5 & Time + coordinate & 0.28 & 0.067 & 0.290 & 0.069 & 0.208 \\
\hline
\end{tabular}

\subsection{Decision making Diagram}

The decision making diagram has been created based on the normalized matrix of relative weights. This diagram has been shown in figure 1 .

\subsection{Deter mination of composite weight of each choice}

The composite weight will be computed by equation (3).

$$
U_{\mathrm{m}}=\sum_{\mathrm{i}=1}^{\mathrm{n}} \mathrm{w}_{\mathrm{i}} \mathrm{w}_{\mathrm{m}, \mathrm{i}}
$$

In this equation $\mathrm{m}$ is the number of choice which its composite weight is under computation.

The computed composite weight of choices has been summarized in table 5 .

Table 5: the composite weight

\begin{tabular}{|c|c|c|}
\hline No. & Control Method & Composite weight $\left(\mathrm{U}_{\mathrm{m}}\right)$ \\
\hline 1 & Time schedule & 0.220 \\
\hline 2 & Command & 0.227 \\
\hline 3 & Coordinate & 0.160 \\
\hline 4 & Command - time schedule & 0.232 \\
\hline 5 & Time schedule - coordinate & 0.161 \\
\hline
\end{tabular}

\subsection{The final choice de termination based on composite weight}

The composite weight is the final result of computations in the composite method and determination of the optimum choice will be based on this quantity.

Although the results has been summarized in the table 5 but in order to make the decision making process more sensible the composite weights have been shown in a column chart in figure 2 . 
The results of AHP method is similar to the control method which had been predicted upon the experts' experience [12].

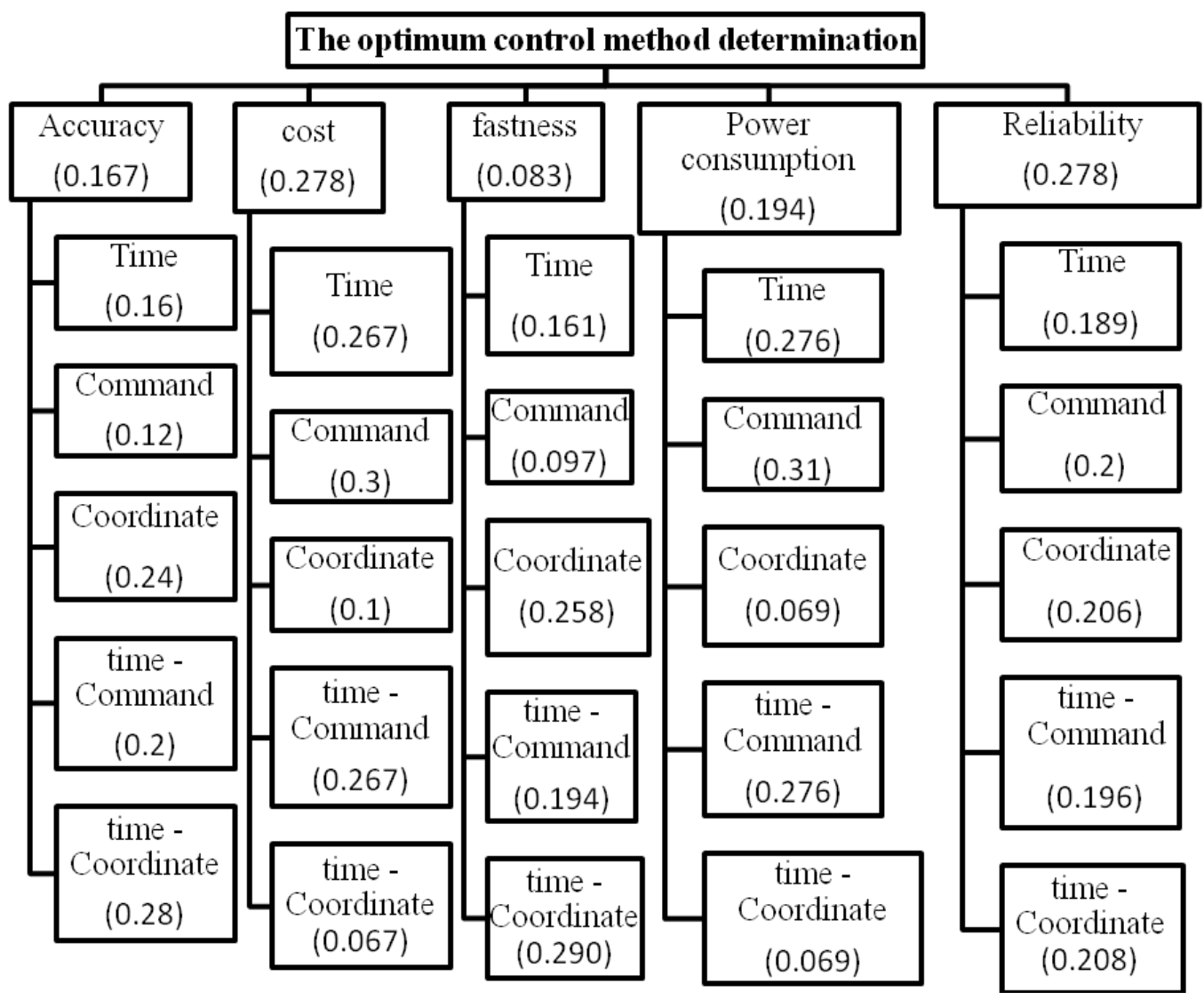

Figure 1: Decision making diagram based on AHP method

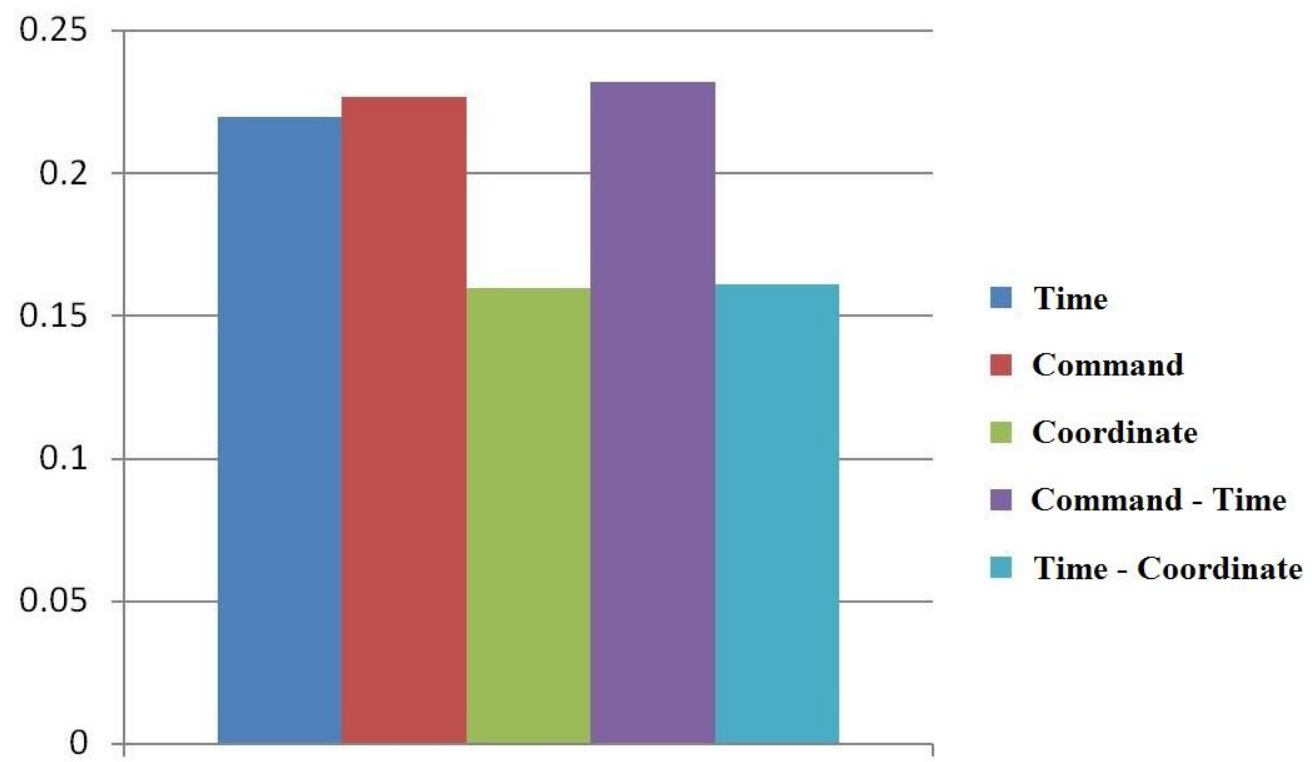

Figure 2: the satellite control methods comparison based on their composite weight 


\section{Conclusion}

In this article the satellite control methods has been classified. Although these methods, are used individually or combined in the space systems but they had not been classified as presented in this paper. In this article all satellite control methods including command - based, time schedule and coordinate based control methods and the ir combinations (combined methods) has been explained completely. The manner of implementation, respective requirements and advantages and disadvantages of the control methods have been studied. When the system engineers based on the users' and mission operations requirements, have more than one choice between the satellite control methods they should use a reasonable manner to choose one of them. The AHP is one of the prevalent methods that help the decision makers to do the best selection among more than one option. An important point in using this method is that the criteria which the selection will be made based on them should have no overlapping with each other. Since in the case has been studied in this article, the criteria overlapped each other, then some of them were deleted and the AHP method started with just five criteria. It must be mentioned that the weights assigned to each criteria are based on the selected case (a small remote sensing satellite) and they can be changed in the other cases as well as the criteria themselves. The final result and the optimum satellite control method was time schedule - command based control method which was a combined method.

By using the AHP method, the system Engineers can be sure that they have observed all the mission operations restrictions and all the users' and beneficiaries' requirements have been provided and eventually they have done the best selection by choosing the optimum choice.

It is proposed system engineers with different idea to study another cases (e.g. a communication satellite) and compare the results.

\section{REFERENCES}

[1] Abbas Jam alipour, "Low Earth Orbital Satellites for Personal Communication Networks", Artech House, 1998;

[2] Bruce R. Elbert, The Satellite Communication Ground Segment and Earth Station Handbook, Artech House, 2001;

[3] Michael O. Kolawole, "Satellite Communication Engineering”, Marcel Dekker Inc, 2002;

[4] Dave Doody, "Basics of Space Flight”, JPL, 2001;

[5] Bruce R. Elbert, “Introduction to Satellite Communication”, 3rd Edition, ARTECH HOUSE, 2008;

[6] Robert W. Jones, "HANDBOOK ON SATELLITE COMMUNICATIONS (HSC)”;

[7] Tri T. Ha, "Digital Satellite Communications", Second Edition, McGraw-Hill Publishing Company, 1990;

[8] Joseph N. Pelton, Robert J. Oslund, Peter Marshall, "COMMUNICATIONS SATELLITE Global Change Agents”, LAWRENCE ERLBAUM ASSOCIATES, PUBLISHERS, 2004;

[9] "EARTH STATION TECHNOLOGY”, Revision 5, Doc. No.: ESTECH-1999-HNDBK-103, June 1999 ; 
[10] Ray E. Sheriff and Y. Fun Hu, "Mobile Satellite Communication Networks", John Wiley \& Sons Ltd, 2001;

[11] Hamdy A. Taha, “Operations Research, An Introduction”, Prentice-Hall of India, New Delhi, 2003;

[12] “IRS-P6 DATA USER'S MANUAL”, Remote Sensing Data User’s Community, October 2003; 